\title{
General aggregation operators acting on the $L$-fuzzy real line
}

\author{
Pavels Orlovs \\ University of Latvia, Department of Mathematics
}

\begin{abstract}
The paper is devoted to a general aggregation operator acting on $L$-fuzzy real numbers. It is defined as a $t$-norm based extension of an ordinary aggregation operator. The aim of our research is to analyze properties of the general aggregation operator depending on properties of the ordinary aggregation operator and the $t$-norm. By using the general aggregation operator we consider the properties of $t$ norm based operations with $L$-fuzzy real numbers such as addition, maximum, minimum.
\end{abstract}

Keywords: $L$-fuzzy real numbers, aggregation operator, general aggregation operator, $T$-extension

\section{Introduction}

Our paper deals with a notion of the $L$-fuzzy real numbers introduced by B. Hutton [1]. He had defined $L$-fuzzy numbers in the case, when $L$ is the unit interval $[0,1]$, but later some other authors (see e.g. [2], [3],[4]) developed and extended his idea.

The notion of a general aggregation operator acting on fuzzy structures was introduced by A. Takaci in [5]. The general aggregation operator is defined by using a $t$-norm $T$ as a $T$-extension of an ordinary aggregation operator. The aim of our research is to analyze properties of the general aggregation operator $\tilde{A}$ acting on the $L$-fuzzy real numbers depending on properties of the ordinary aggregation operator $A$ and the $t$-norm $T$. In particular we consider such properties as associativity, symmetry, idempotence, existence of a neutral element. By using the extended aggregation operator we consider $t$-norm based operations with the $L$-fuzzy real numbers such as addition, maximum, minimum and investigate their properties.

\section{2. $L$-fuzzy real numbers}

Let $L=\left(L, \wedge, \vee, 0_{L}, 1_{L}\right)$ be a complete and completely distributive lattice, equiped with a $t$-norm $T$, where $0_{L}$ and $1_{L}$ are the least and the greatest elements of $L$.

Definition 2.1 An L-fuzzy real number is a function $z: \mathbb{R} \rightarrow L$ such that

(N1) z is non-increasing:

$$
x_{1} \geq x_{2} \Longrightarrow z\left(x_{1}\right) \leq z\left(x_{2}\right)
$$

(N2) $z$ is bounded:

$$
\bigwedge_{x} z(x)=0_{L}, \bigvee_{x} z(x)=1_{L}
$$

(N3) $z$ is left semi-continuous:

$$
\bigwedge_{t<x} z(t)=z(x) .
$$

The set of all $L$-fuzzy real numbers is called the $L$-fuzzy real line and it is denoted by $\mathbb{R}(L)$. In this paper we consider the extended $L$-fuzzy real line: $\overline{\mathbb{R}}(L)=\mathbb{R}(L) \cup\{\tilde{0}\} \cup\{\tilde{1}\}$, where $\tilde{0} \equiv 0_{L}$ and $\tilde{1} \equiv$ $1_{L}$. The elements $\tilde{0}$ and $\tilde{1}$ are the analogues of the infinite elements of the ordinary real line $(-\infty$ and $+\infty$, respectively). We consider that $\overline{\mathbb{R}}(L)$ consists of functions $z$ defined on $\overline{\mathbb{R}}$.

Operations with $L$-fuzzy real numbers such as addition $\oplus_{T}$ and multiplication by a positive real number $k \in \mathbb{R}_{+}$are defined as following:

$$
\begin{gathered}
\left(z_{1} \oplus_{T} \ldots \oplus_{T} z_{n}\right)(x)= \\
=\bigvee_{x=x_{1}+\ldots+x_{n}} T\left(z_{1}\left(x_{1}\right), \ldots, z_{n}\left(x_{n}\right)\right) \\
\text { and } \\
k z(x)=z\left(\frac{x}{k}\right) .
\end{gathered}
$$

\section{Aggregation operator}

We start with the classical notion of an aggregation operator (see e.g. [6],[7],[8]).

Definition 3.1 A mapping $A: \bigcup_{n} \overline{\mathbb{R}}^{n} \rightarrow \overline{\mathbb{R}}$ is called an aggregation operator if the following conditions hold:

(A1) $A(-\infty, \ldots,-\infty)=-\infty$;

(A2) $A(+\infty, \ldots,+\infty)=+\infty$;

(A3) $\forall x_{1}, \ldots, x_{n}, y_{1}, \ldots, y_{n} \in \overline{\mathbb{R}}$ :

$x_{i} \leq y_{i}, i=1, \ldots, n \Longrightarrow$

$\Longrightarrow A\left(x_{1}, \ldots, x_{n}\right) \leq A\left(y_{1}, \ldots, y_{n}\right)$.

Conditions (A1) and (A2) are called boundary conditions of $A$, but (A3) means the monotonicity of $A$. In [5],[6], [7] the arguments of the aggregation operator $A$ are taken from the interval $[0,1]$. But one can consider the case, when instead of $[0,1]$ an arbitrary closed interval $[a, b] \subset[-\infty,+\infty]$ is used (see e.g. [8], where also an arbitrary subinterval of the extended real line is considered). 
Next we define a general aggregation operator $\tilde{A}$ acting on $L^{X}$, where $L^{X}$ is the set of all $L$-fuzzy subsets of a set $X$ [5]. Let $P_{1}, P_{2}, \ldots, P_{n}$ are $L$-sets, i.e. $P_{i}: X \rightarrow L, i=1, \ldots, n$. We denote the order on $L^{X}$ by $\preceq$, but the least and the greatest elements of this order are denoted respectively by $\tilde{0}$ and $\tilde{1}$.

Definition 3.2 A mapping $\tilde{A}: \bigcup_{n}\left(L^{X}\right)^{n} \rightarrow L^{X}$ is called a general aggregation operator if the following conditions hold:

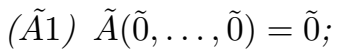

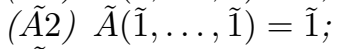

(苂) $\forall P_{1}, \ldots, P_{n}, Q_{1}, \ldots, Q_{n} \in L^{X}$ :

$$
\begin{aligned}
& P_{i} \preceq Q_{i}, i=1, \ldots, n \Longrightarrow \\
& \Longrightarrow \tilde{A}\left(P_{1}, \ldots, P_{n}\right) \preceq \tilde{A}\left(Q_{1}, \ldots, Q_{n}\right) .
\end{aligned}
$$

There exist several approaches to construct a general aggregation operator $\tilde{A}$ based on an ordinary aggregation operator $A$. We use the concept of a $T$-extension of $A$ [5], which idea comes from the classical extension principle (see e.g. [10]). To apply this principle we take $X$ equals to an interval on which $A$ is acting.

Definition 3.3 $\tilde{A}$ is called a $T$-extension of an aggregation operator $A$ if

$$
\begin{gathered}
\tilde{A}\left(P_{1}, \ldots, P_{n}\right)(x)= \\
=\bigvee_{x=A\left(x_{1}, \ldots, x_{n}\right)} T\left(P_{1}\left(x_{1}\right), \ldots, P_{n}\left(x_{n}\right)\right),
\end{gathered}
$$

where $P_{1}, P_{2}, \ldots, P_{n} \in L^{X}, x, x_{1}, x_{2}, \ldots, x_{n} \in X$.

\section{Aggregation of $L$-fuzzy real numbers}

We introduce an aggregation operator on the extended $L$-fuzzy real line $\overline{\mathbb{R}}(L)$ by using the $T$ extension of an ordinary aggregation operator $A$. We assume that $A: \bigcup_{n} \overline{\mathbb{R}}^{n} \rightarrow \overline{\mathbb{R}}$ is a continuous aggregation operator and a $t$-norm $T$ is continuous too. Let us also assume that if $A$ takes the value $+\infty$, then at least one of the arguments is $+\infty$.

We define the operator $\tilde{A}: \bigcup_{n}(\overline{\mathbb{R}}(L))^{n} \rightarrow \overline{\mathbb{R}}(L)$ by the formula

$$
\begin{gathered}
\tilde{A}\left(z_{1}, \ldots, z_{n}\right)(x)= \\
=\bigvee_{x=A\left(x_{1}, \ldots, x_{n}\right)} T\left(z_{1}\left(x_{1}\right), \ldots, z_{n}\left(x_{n}\right)\right),
\end{gathered}
$$

where $z_{1}, z_{2}, \ldots, z_{n} \in \overline{\mathbb{R}}(L), x, x_{1}, x_{2}, \ldots, x_{n} \in \overline{\mathbb{R}}$.

Let us show that the properties $(\tilde{A} 1)-(\tilde{A} 3)$ hold for the operator $\tilde{A}$, then we can be sure that $\tilde{A}$ is a general aggregation operator.

Proof of the property $(\tilde{A} 1)$ :

$$
\begin{gathered}
\tilde{A}(\tilde{0}, \ldots, \tilde{0})(x)= \\
=\bigvee_{x=A\left(x_{1}, \ldots, x_{n}\right)} T\left(\tilde{0}\left(x_{1}\right), \ldots, \tilde{0}\left(x_{n}\right)\right)= \\
=T\left(0_{L}, \ldots, 0_{L}\right)=\tilde{0}(x) .
\end{gathered}
$$

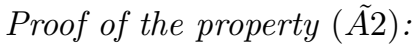

$$
\begin{gathered}
\tilde{A}(\tilde{1}, \ldots, \tilde{1})(x)= \\
\bigvee_{x=A\left(x_{1}, \ldots, x_{n}\right)} T\left(\tilde{1}\left(x_{1}\right), \ldots, \tilde{1}\left(x_{n}\right)\right)= \\
=T\left(1_{L}, \ldots, 1_{L}\right)=\tilde{1}(x) .
\end{gathered}
$$

Proof of the property $(\tilde{A} 3)$ : By the monotonicity of the $t$-norm, for all $x_{1}, \ldots, x_{n}$, we obtain

$$
\begin{gathered}
z_{i} \leq y_{i}, i=1,2, \ldots, n \Longrightarrow \\
T\left(z_{1}\left(x_{1}\right), \ldots, z_{n}\left(x_{n}\right)\right) \leq T\left(y_{1}\left(x_{1}\right), \ldots, y_{n}\left(x_{n}\right)\right) \Longrightarrow \\
\Longrightarrow \bigvee_{x=A\left(x_{1}, \ldots, x_{n}\right)} T\left(z_{1}\left(x_{1}\right), \ldots, z_{n}\left(x_{n}\right)\right) \leq \\
\leq \bigvee_{x=A\left(x_{1}, \ldots, x_{n}\right)} T\left(y_{1}\left(x_{1}\right), \ldots, y_{n}\left(x_{n}\right)\right) \Longrightarrow \\
\Longrightarrow \tilde{A}\left(z_{1}, \ldots, z_{n}\right) \leq \tilde{A}\left(y_{i}, \ldots, y_{n}\right) .
\end{gathered}
$$

Now it is important to show that by using $\tilde{A}$ in the result we get a non-negative $L$-fuzzy real number. It means that we must check the properties $(N 1)$ (N3) for $\tilde{A}\left(z_{1}, \ldots, z_{n}\right)$.

Proposition 4.1 For all $z_{1}, \ldots, z_{n} \in \overline{\mathbb{R}}(L)$ the function $\tilde{A}\left(z_{1}, \ldots, z_{n}\right)$ is non-increasing:

$x_{1} \leq x_{2} \Longrightarrow \tilde{A}\left(z_{1}, \ldots, z_{n}\right)\left(x_{1}\right) \geq \tilde{A}\left(z_{1}, \ldots, z_{n}\right)\left(x_{2}\right)$.

Proof. We have to prove that

$$
\begin{aligned}
& \bigvee_{x_{1}=A\left(\tau_{1}, \ldots, \tau_{n}\right)} T\left(z_{1}\left(\tau_{1}\right), \ldots, z_{n}\left(\tau_{n}\right)\right) \geq \\
\geq & \bigvee_{x_{2}=A\left(t_{1}, \ldots, t_{n}\right)} T\left(z_{1}\left(t_{1}\right), \ldots, z_{n}\left(t_{n}\right)\right) .
\end{aligned}
$$

Let us fix arbitrary $t_{1}, \ldots, t_{n}$ such that

$$
x_{2}=A\left(t_{1}, \ldots, t_{n}\right) .
$$

Taking into account the continuity of $A$ and using the intermediate value theorem, we obtain that

$$
\exists\left(\tau_{1}, \ldots, \tau_{n}\right) \in \prod_{i=1}^{n}\left[-\infty, t_{i}\right]: A\left(\tau_{1}, \ldots, \tau_{n}\right)=x_{1} .
$$

Thereby $\tau_{i} \leq t_{i}$, then $z_{i}\left(\tau_{i}\right) \geq z_{i}\left(t_{i}\right), i=1, \ldots, n$ and

$$
T\left(z_{1}\left(\tau_{1}\right), \ldots, z_{n}\left(\tau_{n}\right)\right) \geq T\left(z_{1}\left(t_{1}\right), \ldots, z_{n}\left(t_{n}\right)\right) .
$$

Then we have

$$
\begin{gathered}
\tilde{A}\left(z_{1}, \ldots, z_{n}\right)\left(x_{1}\right)= \\
=\bigvee_{x_{1}=A\left(u_{1}, \ldots, u_{n}\right)} T\left(z_{1}\left(u_{1}\right), \ldots, z_{n}\left(u_{n}\right)\right) \geq \\
\geq T\left(z_{1}\left(t_{1}\right), \ldots, z_{n}\left(t_{n}\right)\right) .
\end{gathered}
$$

Therefore

$$
\begin{gathered}
\tilde{A}\left(z_{1}, \ldots, z_{n}\right)\left(x_{1}\right) \geq \\
\bigvee_{x_{2}=A\left(t_{1}, \ldots, t_{n}\right)} T\left(z_{1}\left(t_{1}\right), \ldots, z_{n}\left(t_{n}\right)\right)= \\
=\tilde{A}\left(z_{1}, \ldots, z_{n}\right)\left(x_{2}\right) .
\end{gathered}
$$


Proposition 4.2 For all $z_{1}, \ldots, z_{n} \in \overline{\mathbb{R}}(L)$ the function $\tilde{A}\left(z_{1}, \ldots, z_{n}\right)$ is bounded:

$$
\begin{aligned}
& \text { (1) } \bigvee_{x} \tilde{A}\left(z_{1}, \ldots, z_{n}\right)(x)=1_{L}, \\
& \text { (2) } \bigwedge_{x} \tilde{A}\left(z_{1}, \ldots, z_{n}\right)(x)=0_{L} .
\end{aligned}
$$

\section{Proof.}

(1) Taking into account that $\tilde{A}\left(z_{1}, \ldots, z_{n}\right)$ is nonincreasing, we obtain

$$
\begin{gathered}
\bigvee_{x} \bigvee_{x=A\left(x_{1}, \ldots, x_{n}\right)} T\left(z_{1}\left(x_{1}\right), \ldots, z_{n}\left(x_{n}\right)\right) \geq \\
\geq T\left(z_{1}(-\infty), \ldots, z_{n}(-\infty)\right)=1_{L} .
\end{gathered}
$$

(2) By the monotonicity of $\tilde{A}$ :

$$
\begin{aligned}
& \bigwedge_{x} \bigvee_{x=A\left(x_{1}, \ldots, x_{n}\right)} T\left(z_{1}\left(x_{1}\right), \ldots, z_{n}\left(x_{n}\right)\right)= \\
& =\bigvee_{+\infty=A\left(x_{1}, \ldots, x_{n}\right)} T\left(z_{1}\left(x_{1}\right), \ldots, z_{n}\left(x_{n}\right)\right) .
\end{aligned}
$$

The equality $A\left(x_{1}, \ldots, x_{n}\right)=+\infty$ implies that there exists such $i$ that $x_{i}=+\infty$. Then

$$
\begin{gathered}
T\left(z_{1}\left(x_{1}\right), \ldots, z_{n}\left(x_{n}\right)\right)= \\
=T\left(z_{1}\left(x_{1}\right), \ldots, z_{i}(+\infty), \ldots, z_{n}\left(x_{n}\right)\right)=0_{L} .
\end{gathered}
$$

Therefore

$$
\bigwedge_{x} \bigvee_{x=A\left(x_{1}, \ldots, x_{n}\right)} T\left(z_{1}\left(x_{1}\right), \ldots, z_{n}\left(x_{n}\right)\right)=0_{L} .
$$

Proposition 4.3 For all $z_{1}, \ldots, z_{n} \in \overline{\mathbb{R}}(L)$ the function $\tilde{A}\left(z_{1}, \ldots, z_{n}\right)$ is left semi-continuous:

$$
\bigwedge_{x<x_{0}} \tilde{A}\left(z_{1}, \ldots, z_{n}\right)(x)=\tilde{A}\left(z_{1}, \ldots, z_{n}\right)\left(x_{0}\right) .
$$

Proof. Let us denote $\tilde{A}\left(z_{1}, \ldots, z_{n}\right)=z$. Now we have to prove that $\bigwedge_{x<x_{0}} z(x)=z\left(x_{0}\right)$. Let us assume the opposite, i.e.

$$
\bigwedge_{x<x_{0}} z(x)=y\left(x_{0}\right) \neq z\left(x_{0}\right) .
$$

By the monotonicity we have $y\left(x_{0}\right)>z\left(x_{0}\right)$. Let us note, that $x_{0} \neq-\infty$. Basing on the fact that $L$ is completely distributive (see e.g. [9]), we obtain

$$
\begin{gathered}
\bigwedge_{x<x_{0}} \tilde{A}\left(z_{1}, \ldots, z_{n}\right)(x)= \\
=\bigwedge_{x<x_{0}} \bigvee_{x=A\left(t_{1}, \ldots, t_{n}\right)} T\left(z_{1}\left(t_{1}\right), \ldots, z_{n}\left(t_{n}\right)\right)=
\end{gathered}
$$

$$
=\bigvee_{f \in F} \bigwedge_{x<x_{0}} T\left(z_{1}\left(t_{1}^{f, x}\right), \ldots, z_{n}\left(t_{n}^{f, x}\right)\right),
$$

where $F$ is the set of choice functions $f$ such that for all $x<x_{0}$ we have

$$
f(x)=\left(t_{1}^{f, x}, \ldots, t_{n}^{f, x}\right) \text { and } A\left(t_{1}^{f, x}, \ldots, t_{n}^{f, x}\right)=x .
$$

By the assumption

$$
\bigvee_{f \in F} \bigwedge_{x<x_{0}} T\left(z_{1}\left(t_{1}^{f, x}\right), \ldots, z_{n}\left(t_{n}^{f, x}\right)\right)>z\left(x_{0}\right)
$$

one can find such choice function $f$ that

$$
\bigwedge_{x<x_{0}} T\left(z_{1}\left(t_{1}^{f, x}\right), \ldots, z_{n}\left(t_{n}^{f, x}\right)\right) \not \leq z\left(x_{0}\right) .
$$

Let us denote

$$
\alpha\left(x_{0}\right)=\bigwedge_{x<x_{0}} T\left(z_{1}\left(t_{1}^{f, x}\right), \ldots, z_{n}\left(t_{n}^{f, x}\right)\right) .
$$

The value $\alpha\left(x_{0}\right)$ is either greater than $z\left(x_{0}\right)$ or is incomparable with $z\left(x_{0}\right)$.

Now let us take $x=x^{m}$, where

$$
x^{m}=\left\{\begin{array}{l}
x_{0}-\frac{1}{m}, x \neq+\infty, \\
m, x=+\infty
\end{array}\right.
$$

and denote

$t_{i}^{f, x^{m}}=t_{i}^{m}, i=1, \ldots, n, t^{m}=\left(t_{1}^{m}, \ldots, t_{n}^{m}\right), m \in \mathbb{N}$.

The sequence $\left(t^{m}\right)_{m \in \mathbb{N}}$ is such that

$$
T\left(z_{1}\left(t_{1}^{m}\right), \ldots, z_{n}\left(t_{n}^{m}\right)\right) \geq \alpha\left(x_{0}\right) .
$$

We select a subsequence $\left(t^{m_{k}}\right)_{k \in \mathbb{N}}$, which has the limit, and denote this limit by $t^{0}: \lim _{k \rightarrow \infty} t^{m_{k}}=t^{0}$. By the continuity of $A$ :

$$
\begin{gathered}
A\left(t_{1}^{0}, \ldots, t_{n}^{0}\right)=A\left(\lim _{k \rightarrow \infty} t_{1}^{m_{k}}, \ldots, \lim _{k \rightarrow \infty} t_{n}^{m_{k}}\right)= \\
=\lim _{k \rightarrow \infty} A\left(t_{1}^{m_{k}}, \ldots, t_{n}^{m_{k}}\right)=x_{0} .
\end{gathered}
$$

We consider the set

$D=\left\{\left(t_{1}, \ldots, t_{n}\right) \in \overline{\mathbb{R}}^{n} \mid T\left(z_{1}\left(t_{1}\right), \ldots, z_{n}\left(t_{n}\right)\right) \geq \alpha\left(x_{0}\right)\right\}$

and note the following properties:

- if for all $i \in\{1, \ldots, n\} \tau_{i} \leq t_{i}$, then $\tau \in D$;

- if a point $\tau=\left(\tau_{1}, \ldots, \tau_{n}\right)$ is such that $A\left(\tau_{1}, \ldots, \tau_{n}\right) \geq x_{0}$, then $\tau \notin D$.

Let us consider a line $K$ in the the product $\left.\left.\prod_{i=1}^{n}\right]-\infty, t_{i}^{0}\right]$, excluding point $t^{0}$. Such a line can be described by the equations $\left.\left.t_{i}=\tau_{i}(u), u \in\right]-\infty, 1\right]$ for all $i \in\{1, \ldots, n\}$ :

- if $0<t_{i}^{0}<+\infty$, then $\tau_{i}(u)=u t_{i}^{0}$;

- if $t_{i}^{0} \leq 0$, then $\tau_{i}(u)=(2-u) t_{i}^{0}$;

- if $t_{i}^{0}=+\infty$, then $\tau_{i}(u)=\frac{u}{1-u}$, for all $u \in]-\infty, 1\left[\right.$ and $\tau_{i}(1)=+\infty$. 
Let us note, that $\tau(1)=t^{0}$. Also when $u \rightarrow-\infty$, then $\tau_{i}=-\infty$. All points $\tau=\tau(u)$ of the line $K$, when $u \in]-\infty, 1$, belong to the set $D$ : if we fix some point $\tau^{0} \in K, \tau^{0} \neq t^{0}$, then in every neighborhood of the point $t^{0}$ one can find such a point $t^{m_{k_{0}}}$ from the sequence $\left(t^{m_{k}}\right)_{k \in \mathbb{N}}$ that for all $i \in\{1, \ldots, n\}$ $\tau_{i}^{0}<t_{i}^{m_{k_{0}}}$. Now taking into account that $t^{m_{k_{0}}} \in D$, we get $\tau^{0} \in D$.

For every $m \in \mathbb{N}$ on the line $K$ one can find a point $\tau^{m}$ such that $A\left(\tau_{1}^{m}, \ldots, \tau_{n}^{m}\right)=x_{0}-\frac{1}{m}$. This point can be found by using the continuity of $A$ and the intermediate value theorem. Then

$$
\lim _{m \rightarrow \infty} A\left(\tau_{1}^{m}, \ldots, \tau_{n}^{m}\right)=\lim _{m \rightarrow \infty}\left(x_{0}-\frac{1}{m}\right)=x_{0} .
$$

We choose the convergent subsequence $\left(\tau^{m_{k}}\right)_{k \in \mathbb{N}}$ such that

$$
\begin{gathered}
\lim _{k \rightarrow \infty} \tau^{m_{k}}=t^{0}, \text { since } \lim _{k \rightarrow \infty} \tau^{m_{k}} \in K \text { and } \\
\lim _{k \rightarrow \infty} A\left(\tau_{1}^{m_{k}}, \ldots, \tau_{n}^{m_{k}}\right)=x_{0} .
\end{gathered}
$$

Now let us take the limit in $T\left(z_{1}\left(\tau_{1}^{m_{k}}\right), \ldots, z_{n}\left(\tau_{n}^{m_{k}}\right)\right)$ :

$$
\begin{aligned}
& \bigwedge_{k} T\left(z_{1}\left(\tau_{1}^{m_{k}}\right), \ldots, z_{n}\left(\tau_{n}^{m_{k}}\right)\right)= \\
= & T\left(\bigwedge_{k} z_{1}\left(\tau_{1}^{m_{k}}\right), \ldots, \bigwedge_{k} z_{n}\left(\tau_{n}^{m_{k}}\right)\right)= \\
= & T\left(z_{1}\left(t_{1}^{0}\right), \ldots, z_{n}\left(t_{n}^{0}\right)\right)=z\left(x_{0}\right) .
\end{aligned}
$$

Here we have got a contradiction, since $\bigwedge_{k} T\left(z_{1}\left(\tau_{1}^{m_{k}}\right), \ldots, z_{n}\left(\tau_{n}^{m_{k}}\right)\right) \geq \alpha\left(x_{0}\right)$. Thereby our assumption was wrong and $\bigwedge_{x<x_{0}} z(x)=z\left(x_{0}\right)$ or

$$
\bigwedge_{x<x_{0}} \tilde{A}\left(z_{1}, \ldots, z_{n}\right)(x)=\tilde{A}\left(z_{1}, \ldots, z_{n}\right)\left(x_{0}\right) .
$$

\section{Properties of aggregation operator $\tilde{A}$}

Now we consider some properties of aggregation operator $\tilde{A}$ acting on $L$-fuzzy real numbers. As $\tilde{A}$ is defined by an ordinary aggregation operator $A$ and a $t$-norm $T$, it is natural to investigate the properties of $\tilde{A}$ depending on the properties of $A$ and $T$.

Proposition 5.1 If operator $A$ is associative, then operator $\tilde{A}$ is associative:

$\forall z_{1}, z_{2}, z_{3} \in \overline{\mathbb{R}}(L) \tilde{A}\left(z_{1}, \tilde{A}\left(z_{2}, z_{3}\right)\right)=\tilde{A}\left(\tilde{A}\left(z_{1}, z_{2}\right), z_{3}\right)$.

Proof.

$$
\begin{gathered}
\tilde{A}\left(z_{1}, \tilde{A}\left(z_{2}, z_{3}\right)\right)(x)= \\
=\bigvee_{x=A\left(x_{1}, x_{2}\right)} T\left(z_{1}\left(x_{1}\right), \tilde{A}\left(z_{2}, z_{3}\right)\left(x_{2}\right)\right)=
\end{gathered}
$$

$$
\begin{aligned}
& =\bigvee_{x=A\left(x_{1}, x_{2}\right)} T\left(z_{1}\left(x_{1}\right), \bigvee_{x_{2}=A\left(x_{3}, x_{4}\right)} T\left(z_{2}\left(x_{3}\right), z_{3}\left(x_{4}\right)\right)\right)= \\
& =\bigvee_{x=A\left(x_{1}, x_{2}\right)} \bigvee_{x_{2}=A\left(x_{3}, x_{4}\right)} T\left(z_{1}\left(x_{1}\right), T\left(z_{2}\left(x_{3}\right), z_{3}\left(x_{4}\right)\right)\right)= \\
& =\bigvee_{x=A\left(x_{1}, A\left(x_{3}, x_{4}\right)\right)} T\left(T\left(z_{1}\left(x_{1}\right), z_{2}\left(x_{3}\right)\right), z_{3}\left(x_{4}\right)\right)= \\
& =\bigvee_{\left.x=A\left(A\left(x_{1}, x_{3}\right), x_{4}\right)\right)} T\left(T\left(z_{1}\left(x_{1}\right), z_{2}\left(x_{3}\right)\right), z_{3}\left(x_{4}\right)\right)= \\
& =\bigvee_{x=A\left(x_{2}, x_{4}\right)} \bigvee_{x_{2}=A\left(x_{1}, x_{3}\right)} T\left(T\left(z_{1}\left(x_{1}\right), z_{2}\left(x_{3}\right)\right), z_{3}\left(x_{4}\right)\right)= \\
& =\bigvee_{x=A\left(x_{2}, x_{4}\right)} T\left(\bigvee_{x_{2}=A\left(x_{1}, x_{3}\right)} T\left(z_{1}\left(x_{1}\right), z_{2}\left(x_{3}\right)\right), z_{3}\left(x_{4}\right)\right)= \\
& =\bigvee_{x=A\left(x_{2}, x_{4}\right)} T\left(\tilde{A}\left(z_{1}, z_{2}\right)\left(x_{2}\right), z_{3}\left(x_{4}\right)\right)= \\
& =\tilde{A}\left(\tilde{A}\left(z_{1}, z_{2}\right), z_{3}\right)(x) .
\end{aligned}
$$

Proposition 5.2 If operator $A$ is commutative, then operator $\tilde{A}$ is commutative:

$$
\forall z_{1}, z_{2} \in \overline{\mathbb{R}}(L) \quad \tilde{A}\left(z_{1}, z_{2}\right)=\tilde{A}\left(z_{2}, z_{1}\right) .
$$

\section{Proof.}

$$
\begin{aligned}
& \tilde{A}\left(z_{1}, z_{2}\right)(x)=\bigvee_{x=A\left(x_{1}, x_{2}\right)} T\left(z_{1}\left(x_{1}\right), z_{2}\left(x_{2}\right)\right)= \\
& =\bigvee_{x=A\left(x_{2}, x_{1}\right)} T\left(z_{2}\left(x_{2}\right), z_{1}\left(x_{1}\right)\right)=\tilde{A}\left(z_{2}, z_{1}\right)(x) .
\end{aligned}
$$

Proposition 5.3 If operator $A$ is idempotent and $T$ is a minimum t-norm $T_{M}$, then operator $\tilde{A}$ is idempotent:

$$
\forall z \in \overline{\mathbb{R}}(L) \quad \tilde{A}(z, \ldots, z)=z .
$$

Proof.

First of all let us show that $\tilde{A}(z, \ldots, z)(x) \geq z(x)$ :

$$
\begin{gathered}
\tilde{A}(z, \ldots, z)(x)=\bigvee_{x=A\left(x_{1}, \ldots, x_{n}\right)} T_{M}\left(z\left(x_{1}\right), \ldots, z\left(x_{n}\right)\right) \geq \\
\geq \bigvee_{x=A(x, \ldots, x)} T_{M}(z(x), \ldots, z(x))=z(x) .
\end{gathered}
$$

Now we should prove that $\tilde{A}(z, \ldots, z)(x) \leq z(x)$. For an ordinary aggregation operator $A$ the idempotence is equivalent to the compensation property (see e.g. [7]):

$$
\min \left(x_{1}, \ldots, x_{n}\right) \leq A\left(x_{1}, \ldots, x_{n}\right) \leq \max \left(x_{1}, \ldots, x_{n}\right) .
$$

Using this property we obtain

$$
x=A\left(x_{1}, \ldots, x_{n}\right) \Longrightarrow x \leq \max \left(x_{1}, \ldots, x_{n}\right) \Longrightarrow
$$




$$
\Longrightarrow z(x) \geq T_{M}\left(z\left(x_{1}\right), \ldots, z\left(x_{n}\right)\right) .
$$

Therefore

$$
\begin{gathered}
z(x) \geq \bigvee_{x=A\left(x_{1}, \ldots, x_{n}\right)} T_{M}\left(z\left(x_{1}\right), \ldots, z\left(x_{n}\right)\right)= \\
=\tilde{A}(z, \ldots, z)(x) .
\end{gathered}
$$

\section{Proposition 5.4 The absorbing element of op-} erator $\tilde{A}$ is element $\tilde{0}$ :

$$
\tilde{A}\left(z_{1}, \ldots, z_{i-1}, \tilde{0}, z_{i+1}, \ldots, z_{n}\right)=\tilde{a} .
$$

\section{Proof.}

$$
\begin{gathered}
\tilde{A}\left(z_{1}, \ldots, z_{i-1}, \tilde{0}, z_{i+1}, \ldots, z_{n}\right)(x)= \\
=\bigvee_{x=A\left(x_{1}, \ldots, x_{n}\right)} T\left(z_{1}\left(x_{1}\right), \ldots, \tilde{0}\left(x_{i}\right), \ldots, z_{n}\left(x_{n}\right)\right)= \\
=\bigvee_{x=A\left(x_{1}, \ldots, x_{n}\right)} T\left(z_{1}\left(x_{1}\right), \ldots, 0_{L}, \ldots, z_{n}\left(x_{n}\right)\right)=0_{L} .
\end{gathered}
$$

Proposition 5.5 If operator $A$ is homogeneous by means of multiplication with a positive real number, then $\tilde{A}$ is homogeneous by means of multiplication with a positive real number:

$$
\tilde{A}\left(k z_{1}, \ldots, k z_{n}\right)=k \tilde{A}\left(z_{1}, \ldots, z_{n}\right), \quad k>0 .
$$

Proof.

$$
\begin{gathered}
={ } \tilde{A}\left(k z_{1}, \ldots, k z_{n}\right)(x)= \\
=\bigvee_{x=A\left(x_{1}, \ldots, x_{n}\right)} T\left(k z_{1}\left(x_{1}\right), \ldots, k z_{n}\left(x_{n}\right)\right)= \\
=\bigvee_{x=A\left(x_{1}, \ldots, x_{n}\right)} T\left(z_{1}\left(\frac{x_{1}}{k}\right), \ldots, z_{n}\left(\frac{x_{n}}{k}\right)\right)= \\
=\bigvee_{\frac{x}{k}=\frac{1}{k} A\left(x_{1}, \ldots, x_{n}\right)} T\left(z_{1}\left(\frac{x_{1}}{k}\right), \ldots, z_{n}\left(\frac{x_{n}}{k}\right)\right)= \\
=\bigvee_{\frac{x}{k}=A\left(\frac{x_{1}}{k}, \ldots, \frac{x_{n}}{k}\right)} T\left(z_{1}\left(\frac{x_{1}}{k}\right), \ldots, z_{n}\left(\frac{x_{n}}{k}\right)\right)= \\
=\tilde{A}\left(z_{1}, \ldots, z_{n}\right)\left(\frac{x}{k}\right)=k \tilde{A}\left(z_{1}, \ldots, z_{n}\right)(x) .
\end{gathered}
$$

\section{Operations with $L$-fuzzy real numbers}

In this section we consider such $t$-norm based operations with $L$-fuzzy real numbers as addition, minimum and maximum. We can rewrite the formula of addition by using the general aggregation operator of arithmetic mean $\widetilde{A}_{M}$, which is based on the ordinary aggregation operator of arithmetic mean $A_{M}$ :

$$
z_{1} \oplus_{T} \ldots \oplus_{T} z_{n}=n \tilde{A}_{M}\left(z_{1}, \ldots, z_{n}\right) .
$$

The ordinary aggregation operator is defined as the function $A_{M}: \overline{\mathbb{R}}^{n} \rightarrow \overline{\mathbb{R}}$ by the following formula:

$$
A_{M}\left(t_{1}, t_{2}, \ldots, t_{n}\right)=\frac{1}{n} \sum_{i=1}^{n} t_{i}, \quad t_{1}, t_{2}, \ldots, t_{n} \in \overline{\mathbb{R}} .
$$

We assume that if at least one of the arguments of $A_{M}$ is $+\infty$, then $A_{M}$ takes the value $+\infty$.

The formula for $\oplus_{T}$ is an equivalent of the classical formula for addition of $L$-fuzzy real numbers:

$$
\begin{gathered}
n \tilde{A}_{M}\left(z_{1}, \ldots, z_{n}\right)(x)=\tilde{A}_{M}\left(z_{1}, \ldots, z_{n}\right)\left(\frac{x}{n}\right)= \\
=\bigvee_{\frac{x}{n}=A_{M}\left(x_{1}, \ldots, x_{n}\right)} T\left(z_{1}\left(x_{1}\right), \ldots, z_{n}\left(x_{n}\right)\right)= \\
\quad=\bigvee_{x=x_{1}+\ldots+x_{n}} T\left(z_{1}\left(x_{1}\right), \ldots, z_{n}\left(x_{n}\right)\right) .
\end{gathered}
$$

Some properties of the operator $\oplus_{T}$ can be obtained from the corresponding properties of $\tilde{A}_{M}$. As the ordinary $A_{M}$ operator is associative, commutative, and homogeneous, the operator $\oplus_{T}$ is associative, commutative and homogeneous as well.

The property of distributivity

$$
(\alpha+\beta) z=\alpha z \oplus_{T} \beta z, \quad \alpha, \beta>0
$$

does not hold for an arbitrary $t$-norm. For example, in the case of product $t$-norm $T_{P}: z \oplus_{T_{P}} z \neq 2 z$ for some $z \in \overline{\mathbb{R}}(L)$. The distributivity holds, when the extension of operator $A_{M}$ preserves the idempotence. Really, taking into account the equality

$$
\tilde{A}_{M}((\alpha+\beta) z,(\alpha+\beta) z)=\tilde{A}_{M}(2 \alpha z, 2 \beta z),
$$

which holds in the case of minimum $t$-norm $T_{M}$, the distributivity can be reduced to

$$
\tilde{A}_{M}((\alpha+\beta) z,(\alpha+\beta) z)=(\alpha+\beta) z
$$

(this last equality means the idempotence property). To prove the equality we consider

$$
\begin{gathered}
\tilde{A}_{M}((\alpha+\beta) z,(\alpha+\beta) z)(x)= \\
=\bigvee_{x_{1}+x_{2}=2 x} T_{M}\left(z\left(\frac{x_{1}}{\alpha+\beta}\right), z\left(\frac{x_{2}}{\alpha+\beta}\right)\right)= \\
=z\left(\frac{x}{\alpha+\beta}\right), \\
\tilde{A}_{M}(2 \alpha z, 2 \beta z)(x)= \\
=\bigvee_{x_{1}+x_{2}=2 x} T_{M}\left(z\left(\frac{x_{1}}{2 \alpha}\right), z\left(\frac{x_{2}}{2 \beta}\right)\right)=z\left(x_{0}\right),
\end{gathered}
$$

where $x_{0}=\frac{x}{\alpha+\beta}$ is obtained as the solution of the following system of linear equations:

$$
\left\{\begin{array}{l}
\frac{x_{1}}{2 \alpha}=\frac{x_{2}}{2 \beta}=x_{0}, \\
x_{1}+x_{2}=2 x .
\end{array}\right.
$$


We consider the operations of minimum and maximum of $L$-fuzzy real numbers by the following formulas:

$$
\begin{gathered}
\operatorname{MIN}\left(z_{1}, \ldots, z_{n}\right)(x)= \\
=\bigvee_{x=\min \left(x_{1}, \ldots, x_{n}\right)} T\left(z_{1}\left(x_{1}\right), \ldots, z_{n}\left(x_{n}\right)\right), \\
\operatorname{MAX}\left(z_{1}, \ldots, z_{n}\right)(x)= \\
=\bigvee_{x=\max \left(x_{1}, \ldots, x_{n}\right)} T\left(z_{1}\left(x_{1}\right), \ldots, z_{n}\left(x_{n}\right)\right) .
\end{gathered}
$$

As the ordinary operators of minimum and maximum are associative, commutative, homogeneous and idempotent, the extended operations $M I N$ and $M A X$ with $L$-fuzzy real numbers will be associative, commutative, homogeneous and idempotent (in the case of minimum $t$-norm) as well. But it is worth to mention that the result of these operations depends on the choice of the $t$-norm. For example, the result of MIN operation in the case of minimum $t$-norm $T_{M}$ will be just the ordinary minimum of functions, but the result in the case of product $t$-norm $T_{P}$ can be different from the ordinary one.

\section{Acknowledgement}

This work has been supported by the European Social Fund within the project «Support for Doctoral Studies at University of Latvia».

\section{References}

[1] B. Hutton, Normality in Fuzzy Topological Spaces, emphJ.Math.Anal.Appl., 50:74-79, 1975.

[2] R. Lowen, On $(\mathbb{R}(L), \oplus)$, Fuzzy Sets and Systems, 10:203-209, 1983.

[3] S. E. Rodabaugh, Fuzzy Addition and the Fuzzy Real Lines, Fuzzy Sets and Systems, 8:39-52, 1982.

[4] S. E. Rodabaugh, Complete Fuzzy Topological Hyperfields and Fuzzy Multiplication in the Fuzzy Real Lines, Fuzzy Sets and Systems, 15:285-310, 1988.

[5] A. Takaci, General Aggregation Operators Acting on Fuzzy Numbers Induced by Ordinary Aggregation Operators, Novi Sad J. Math., 33(2):67-76, 2003.

[6] M.Detyniecki, Fundamentals on Aggregation Operators, Berkeley, 2001.

[7] T. Calvo, G. Mayor, R. Mesiar, Aggregation Operators, Physical-Verlag, Heidelberg, 2002.

[8] M. Grabisch, J.-L. Marichal, R. Mesiar, E. Pap, Aggregation Functions (Encyclopedia of Mathematics and its Applications), Cambridge University Press, 2009.

[9] B. A. Davey, H. A. Priestley, Introduction to Lattices and Order, Cambridge University Press, Second Edition, 2002.

[10] E. P. Klement, R. Mesiar, E. Pap, Triangular Norms, Kluwer Academic Publishers, Dordrecht, 2000. 